\title{
Uso y fuentes de información de métodos anticonceptivos en los estudiantes de las Facultades de Enfermería y Derecho
}

Ríos-Olguín María José, López-Hurtado Balkis de Guadalupe**, Peza-Cruz Gabriela***, Ángel-Padilla Margarita***, Mejía-Trejo María Rosalba****, Zamarripa-Solorio Ixchel Cinteott $^{* * * *}$

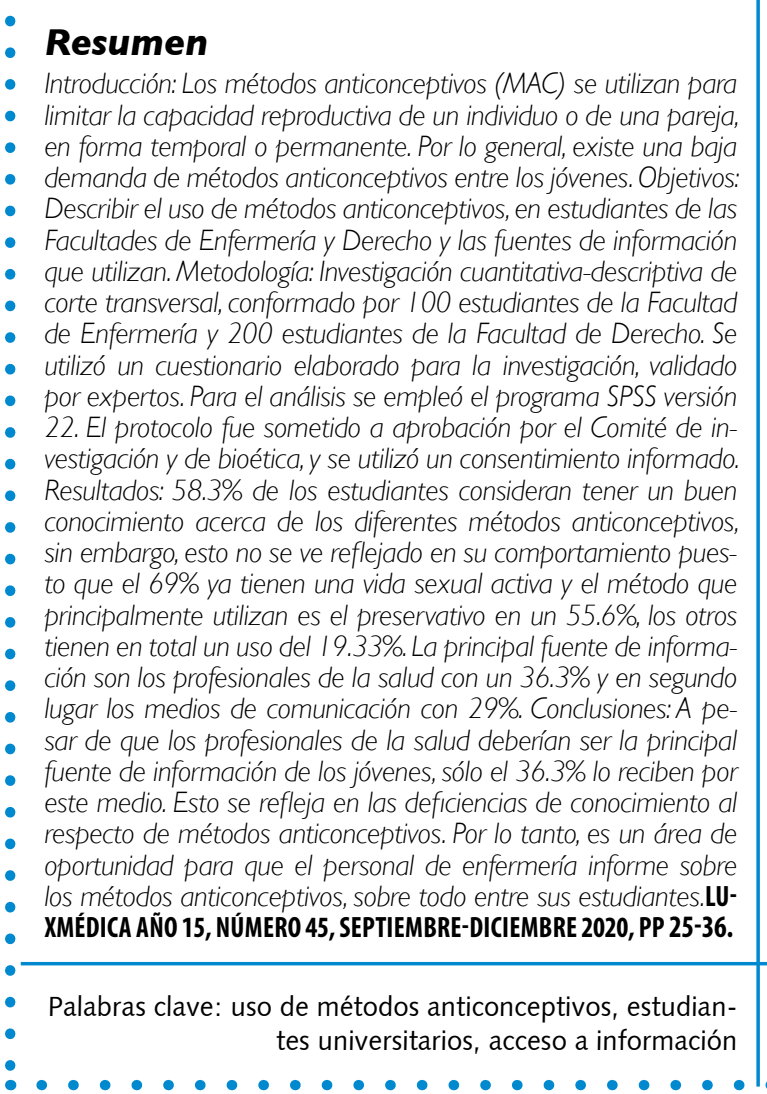

\section{Abstract}

Introduction: Contraceptive methods (CM) are used to limit the reproductive capacity of an individual or a couple, temporarily or permanently. Generally, there is a low demand for contraception among young people. Objective: To describe the use of contraceptive methods in students of the Faculties of Nursing and Law and the sources of information they use. Methods: This is a quantitative-descriptive, cross-sectional study, including 100 students from the Faculty of Nursing and 200 students from the Faculty of Law. A questionnaire elaborated for this research was used, which was validated by experts. For the analysis, the SPSS version 22 program was used. It was submitted to approval by the Research and Bioethics Committee; an informed consent was used. Results: $58.3 \%$ of the students consider that they have good knowledge about the different contraceptive methods; however, this is not reflected in their behavior since $69 \%$ already have an active sexual life and the method they mainly use is the condom in a 55.6\%; the others have total use of $19.33 \%$. The main source of information is health professionals with 36.3\%, and secondly, the media with 29\%. Conclusions: Although health professionals should be the main source of information for young people, only $36.3 \%$ receive it from them. This is reflected in the deficiencies in knowledge regarding contraceptive methods. Therefore, it is an area of opportunity for nursing staff to inform about contraceptive methods, especially among their students. LUXMÉDICA AÑO 15, NÚMERO 45, SEPTIEMBRE-DICIEMBRE 2020, PP 2536.

Keywords: use of contraceptive methods, university students, access to information

Rios-Olguín María José. https:/orcidorg/0000-0003-3610-0399 Pasante de la Licenciatura de Enfermería. Facultad de Enfermería, Universidad Autónoma de Querétaro. Campus San Juan del Río. Correo electrónico: marijjoro16@gmail.com López-Hutada Balkis de Guadalupe. hitps:/orcidorg/0000-0002-9807-3990 Maestra en Nutrición Clinica. Docente en La Facultad de Enfermería, Universidad Autónoma de Querétaro. Campus San Juan del Ría. Correo electrónico: balkis. deguadalupelopez@uaq.mx

*** Peza-Cruz Gabriela. https://orid.org/0000-0002-2995-345X Maestra en Ciencias de la Enfermería. Docente en la Facultad de Enfermería. Universidad Autónoma de Querétaro. Campus San Juan del Río. Correo electrónico: gabrielapeza@ uaa.mx

**** Angel-Padilla Marganita hittps://orcidorg 0000-0001-7165-9521 Licenciatura en Enfermería. Facultad de Enfermería, Universidad Autónoma de Querétaro Campus San Juan del Río. Correo electrónico: angel_padilla marganita@ @hotmail.com

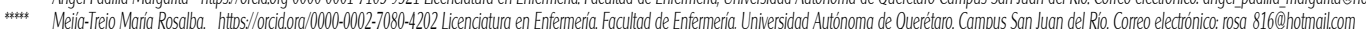

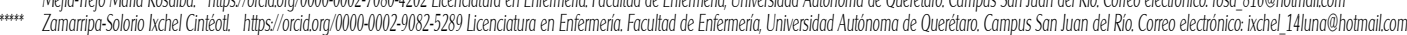

Fecha de recibido 11 de octubre de 2019

Fecha de aceptación: 11 de febrero de 2020

Correspondencia: Balkis de Guadalupe López Hutado. Universidad Autónoma de Querétaro, Facultad de Enfermería, Campus San Juan del Río. Domicilio: Avenida Río Moctezuma No. 249, sección 8, colonia San Cayetano San Juan del Río Querétaro. Código Postal: 76807. Facultad de Enfermería. Tel: 427-2743168. Correo electrónico: balkis.deguadalupelopez@uaq.mx 


\section{Introducción}

Actualmente existen métodos anticonceptivos (MAC) que están a la disposición de la población y por ser un programa prioritario del sector salud, se pueden obtener de manera gratuita en los distintos centros de salud de México; sin embargo, existen algunos factores que disminuyen o limitan su uso. Las mujeres que no utilizan algún método tienen un $90 \%$ de posibilidad de tener un embarazo no deseado. ${ }^{1}$ De acuerdo con la NOM 005-SSA2-1993, los MAC son aquellos que se utilizan para delimitar la capacidad reproductiva de un individuo o de una pareja, en forma temporal o permanente. ${ }^{2}$ Cada mujer puede decidir cuál MAC es el que resuelve su necesidad anticonceptiva, siempre y cuando esté bien informada al respecto, teniendo en cuenta sus condiciones personales, estado de salud, y frecuencia de relaciones sexuales. Actualmente, existen diferentes MAC eficaces si son usados correctamente; además, son accesibles y están a la disposición de las parejas para evitar embarazos no deseados $y$, algunos, ayudan a prevenir infecciones de transmisión sexual (ITS). ${ }^{3}$ La decisión de cuál MAC es el adecuado para cada pareja, requiere de una valoración entre las ventajas y las desventajas de los diferentes métodos. Los factores que se deben tener en cuenta al elegir un método anticonceptivo, incluyen las características de la persona, el perfil de efectos adversos de los distintos productos, el costo, la disponibilidad y las preferencias de las personas. ${ }^{4}$

De acuerdo con la NOM 005-SSA2-1993 los métodos anticonceptivos temporales pueden ser hormonales y previenen el embarazo mediante la inhibición total o parcial de la ovulación. Estos métodos brindan protección anticonceptiva del 90 al 99\%. Pueden ser administrados vía oral, inyectables, implante subdérmico, parche transdérmico o pastillas de anticoncepción de emergencia (PAE). ${ }^{1}$ Considerados también como métodos temporales se encuentran los de barrera que actúan evitando la fecundación de manera mecánica. Algunos de ellos tienen la capacidad de prevenir el contagio de ITS hasta en un $97 \%$ y brindan una protección anticonceptiva del 85 al 97\%, por lo general no se presentan efectos colaterales. Dentro de este método se encuentran los siguientes: preservativo masculino, preservativo femenino, espermicida, diafragma, dispositivo intrauterino (DIU). ${ }^{2}$ En cuanto a los MAC definitivos o irreversibles, son los que impiden la capacidad reproductiva de forma permanente, que se lleva a cabo mediante un procedimiento quirúrgico al hombre o a la mujer. Dentro de estos métodos se encuentran: oclusión tubárica bilateral (OTB) y vasectomía. ${ }^{5}$ Hoy en día el no utilizar ningún método anticonceptivo se considera como un problema de la salud pública, debido al incremento de embarazos no deseados e ITS en los adultos jóvenes.

Con base en lo anterior, es importante resaltar que la maternidad no planeada se ha convertido en una de las causas principales de deserción universitaria. ${ }^{6}$ En México, durante los últimos 15 años 
el índice de deserción universitaria ha sido reportada entre $7.5 \%$ y $8.5 \%$ en la escala nacional, siendo una de las causas el embarazo no deseado, por la adquisición de nuevas responsabilidades como la crianza. ${ }^{7}$ A pesar de la información recibida, aún hay carencia entre los jóvenes de ejercer una vida sexual activa responsablemente, lo que propicia la existencia de embarazos no deseados y el riesgo de contraer alguna ITS a temprana edad, que puede conducir a la deserción escolar o el abandono académico temprano. ${ }^{8}$

Las infecciones de transmisión sexual (ITS) por su parte, son un conjunto de infecciones ocasionadas por diferentes agentes etiológicos que se adquieren por vía sexual, existiendo la posibilidad de generar infecciones diseminadas lesionando otros órganos. ${ }^{9-10}$ De acuerdo con la Organización Mundial de la Salud, a nivel mundial se estima que existen 357 millones de personas que contraen alguna ITS, entre las más comunes se encuentran: clamidiasis con 131 millones, gonorrea 78 millones, sífilis 5,6 millones y tricomoniasis 143 millones. En el sexo masculino la ITS con mayor prevalencia es el virus de herpes simple (VHS), superando los 500 millones de hombres infectados y en las mujeres se registra el virus del papiloma humano (VPH) con una prevalencia de 290 millones de casos. ${ }^{11}$

En México, la información sobre los métodos anticonceptivos en el año 2014 indicó que el 95\% de los jóvenes refirieron haber escuchado y conocer al menos un método anticonceptivo, sin embargo, sólo el $37 \%$ de la población joven en el país utilizó alguno de ellos en su primera relación sexual. ${ }^{12}$

El Instituto Nacional de Estadística y Geografía, ${ }^{13}$ refiere que dentro de los métodos más empleados se encuentra el preservativo utilizado en un $7.2 \%$, las pastillas anticonceptivas con un uso del $5.4 \%$, la inyección con $3.9 \%$, el implante subdérmico en $1.5 \%$ y por último el parche con $0.7 \%$. De acuerdo con la Encuesta Nacional de Salud y Nutrición (ENSANUT) ${ }^{14}$ en el estado de Querétaro el uso de métodos anticonceptivos en los jóvenes fue de $64.3 \%$, en cambio en el municipio de San Juan del Río la cantidad de métodos proporcionados a los jóvenes en el año 2014-2015 fue en total de $75 \%$, esto de acuerdo con los datos otorgados por la jurisdicción sanitaria $\mathrm{N}^{\circ} 2$.

Por consiguiente, se considera importante la educación sexual en los jóvenes, para disminuir los embarazos no deseados e ITS 9 . Se hace mención de lo anterior, ya que el método anticonceptivo más utilizado entre los jóvenes universitarios es el preservativo, cuando lo ideal es el uso simultáneo de preservativos con otros métodos anticonceptivos para disminuir la posibilidad de adquirir una ITS. ${ }^{4}$ El usar sólo el preservativo tiene hasta un $13 \%$ de riesgo para presentar embarazo y hasta $10 \%$ de riesgo de adquirir una ITS. ${ }^{11}$ El objetivo general de la presente investigación fue describir el uso de métodos anticonceptivos, en estudiantes de las Facultades de Enfermería y Derecho de una Universidad Pública y las fuentes de 
información que utilizan los estudiantes. Se seleccionó la Facultad de Enfermería debido a que es una profesión dirigida al cuidado integral del ser humano y es una carrera que en su carga curricular aborda materias donde se habla sobre métodos anticonceptivos e ITS.

Esta investigación surge como un referente empírico basado en la observación en las distintas ferias de la salud organizadas por ENSAIN donde se evidencian una mayor participación en las pláticas sobre salud sexual y reproductiva y mayor demanda de anticonceptivos hormonales y de barrera por los estudiantes de Derecho que por los de Enfermería.

| | | | | | | | | | | | | | | | | | | | | | | | | | | | | | | | | | | | | | | | | | | | | | | | | | | | | | | | | | | | | | | | | | | | | | | | | | | | | | | | | | | | | | | | | | | | | | | | | | ||

\section{Material y métodos}

El presente estudio tuvo un enfoque cuantitativo, con diseño descriptivo y de corte trasversal. El universo estuvo conformado por 112 estudiantes de la Facultad de Enfermería y 355 estudiante de la Facultad de Derecho de la Universidad Autónoma de Querétaro, Campus San Juan del Río en el periodo 2016 - 2017, que además cubriera los criterios de selección. El tamaño de la muestra se calculó a través de fórmula estadística para poblaciones finitas, y se procedió a realizar el muestreo aleatorio estratificado, de los cuales 12 de Enfermería y 155 de Derecho no cumplieron con los criterios de selección, quedando una muestra definitiva de 100 alumnos de Enfermería y 200 de Derecho. La unidad de observación fueron estudiantes de 18-25 años, inscritos en las Facultades de Enfermería y Derecho, en el periodo 2017-2. Se eliminaron a los alumnos que no firmaron el consentimiento informado, con cuestionario no resuelto como mínimo en un $80 \%$ y abandono de la investigación. Se obtuvo la autorización por parte del Subcomité de Investigación y Comité de Ética de la Facultad de Enfermería. El cuestionario de recolección de datos que se aplicó fue elaborado ex profeso para la investigación denominado "Aspectos que influyen en el uso de métodos anticonceptivos en estudiantes universitarios" y para su elaboración se tomaron en cuenta dos instrumentos, el primer instrumento es Universidad de Castilla-La Mancha, que lleva por título Cuestionario Sobre Sexualidad y Anticoncepción en Jóvenes Universitarios de Enfermería, ${ }^{6}$ y el segundo se titula Factores Asociados que influyen en el inicio de actividad sexual en adolescentes escolares de Villa San Francisco-Santa Anita de la Universidad Federico Villareal en Lima Perú. ${ }^{15}$ El cuestionario fue validado a través de expertos y mediante prueba piloto. Se elaboró una base de datos en el

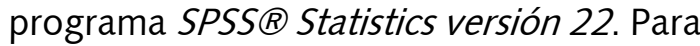
el análisis estadístico se utilizó estadística descriptiva.

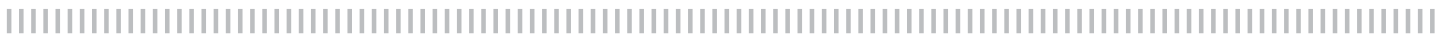

\section{Resultados}

A continuación, se muestran los resultados de las variables de estudio. La muestra final fue conformada por 200 estudiantes de la Facultad de Derecho y 100 estudiantes de la Facultad de Enfermería, adscritos en el periodo escolar agosto-diciembre 2017-2 de la Universidad Pública. La mayoría de los encuestados pertenecen al rango de edad de 18 a 21 años; en ambos grupos predominó el sexo femenino, y como estado civil la mayoría manifestó ser soltero. Más de la mitad tienden a llevar una vida sexual activa (tabla 1). 


\section{Tabla I}

Datos sociodemográficos de los estudiantes universitarios participantes

\begin{tabular}{|c|c|c|c|c|c|}
\hline \multirow{2}{*}{\multicolumn{2}{|c|}{ Datos sociodemográficos }} & \multicolumn{2}{|c|}{ Enfermería } & \multicolumn{2}{|c|}{ Derecho } \\
\hline & & \multirow{2}{*}{$\begin{array}{c}\mathbf{f} \\
71 \\
29\end{array}$} & \multirow{2}{*}{$\begin{array}{l}\% \\
71 \\
29\end{array}$} & \multirow{2}{*}{$\begin{array}{c}\mathbf{f} \\
149 \\
51\end{array}$} & \multirow{2}{*}{$\begin{array}{c}\% \\
74.5 \\
25.5\end{array}$} \\
\hline $\begin{array}{l}\text { Edad } \\
\text { (Años) }\end{array}$ & $\begin{array}{l}18 \text { a } 21 \\
22 \text { a } 25\end{array}$ & & & & \\
\hline Sexo & $\begin{array}{l}\text { Femenino } \\
\text { Masculino }\end{array}$ & $\begin{array}{l}85 \\
15\end{array}$ & $\begin{array}{l}85 \\
15\end{array}$ & $\begin{array}{c}118 \\
82\end{array}$ & $\begin{array}{l}59 \\
41\end{array}$ \\
\hline Estado civil & $\begin{array}{l}\text { Separado } \\
\text { Unión libre } \\
\text { Casado } \\
\text { Soltero }\end{array}$ & $\begin{array}{c}1 \\
4 \\
4 \\
91\end{array}$ & $\begin{array}{c}1 \\
4 \\
4 \\
91\end{array}$ & $\begin{array}{c}9 \\
5 \\
4 \\
182\end{array}$ & $\begin{array}{c}4.5 \\
2.5 \\
2 \\
91\end{array}$ \\
\hline Hijos & $\begin{array}{l}\text { Sí } \\
\text { No }\end{array}$ & $\begin{array}{c}8 \\
92\end{array}$ & $\begin{array}{c}8 \\
92\end{array}$ & $\begin{array}{c}23 \\
177\end{array}$ & $\begin{array}{l}11.5 \\
88.5\end{array}$ \\
\hline Vida sexual & $\begin{array}{l}\text { Sí } \\
\text { No }\end{array}$ & $\begin{array}{l}56 \\
44\end{array}$ & $\begin{array}{l}56 \\
44\end{array}$ & $\begin{array}{c}151 \\
49\end{array}$ & $\begin{array}{l}75 \\
25\end{array}$ \\
\hline
\end{tabular}

Fuente: Cuestionario aspectos que intervienen en el uso de métodos anticonceptivos en estudiantes universitarios. $n=300$

De acuerdo a la percepción que cada estudiante tiene sobre el conocimiento de las diferentes opciones anticonceptivas, la mayoría respondió en orden decreciente, que su conocimiento era bueno, aceptable y excelente; sólo el $1.5 \%$ de los estudiantes de la Licenciatura de Derecho manifestó su valoración como mala o inadecuada (tabla 2).

\section{Tabla 2}

\section{Conocimiento sobre métodos anticonceptivos}

\begin{tabular}{|l|c|c|c|c|}
\hline \multirow{2}{*}{ Valoración del conocimiento } & \multicolumn{2}{|c|}{ Enfermería } & \multicolumn{2}{c|}{ Derecho } \\
\hline Mala & $\mathbf{f}$ & $\%$ & $\mathbf{f}$ & $\%$ \\
\hline Aceptable & 0 & 0 & 3 & 1.5 \\
\hline Buena & 24 & 24 & 65 & 32.5 \\
\hline Excelente & 65 & 65 & 110 & 55 \\
\hline
\end{tabular}

Fuente: Cuestionario aspectos que intervienen en el uso de métodos anticonceptivos en estudiantes universitarios. $n=300$

En la tabla 3 se observa que la mayoría de los jóvenes mencionan que sí conocen las consecuencias de no usar métodos anticonceptivos. $98 \%$ de la población así lo demostró en el cuestionario, pero a pesar de conocer las consecuencias, su uso no es frecuente, por lo cual se recomienda que las Facultades promuevan el uso de éstos, y hacer mayor énfasis en la educación sobre los mismos (tabla 3). 


\section{Tabla 3}

\section{Consecuencias de no usar métodos anticonceptivos}

\begin{tabular}{|l|c|c|c|c|}
\hline \multicolumn{1}{|c|}{ Consecuencias } & \multicolumn{2}{|c|}{ Enfermería } & \multicolumn{2}{c|}{ Derecho } \\
\hline \multicolumn{1}{|c|}{} & f & $\%$ & f & $\%$ \\
\hline Ninguno & 1 & 1 & 0 & 0 \\
\hline Dificultad para casarse & 0 & 0 & 4 & 2 \\
\hline Dificultad para estudiar & 0 & 0 & 1 & 0.5 \\
\hline $\begin{array}{l}\text { Infecciones de transmisión sexual y } \\
\text { embarazo no deseado }\end{array}$ & 99 & 99 & 195 & 97.5 \\
\hline
\end{tabular}

Fuente: Cuestionario aspectos que intervienen en el uso de métodos anticonceptivos en estudiantes universitarios. $n=300$

De los métodos anticonceptivos se reportó el preservativo como el más utilizado, en los estudiantes de la Lic de Enfermería $52 \%$, y en los de la Lic en Derecho $57.5 \%$. Con respecto al conocimiento sobre el uso correcto, la mayoría de los participantes presentó uno adecuado, ya que sabían la colocación debida del preservativo; sin embargo, la discrepancia fue notoria en el uso de la pastilla de emergencia, en donde menos de la mitad de los estudiantes de derecho respondieron correctamente el momento adecuado para ingerir la pastilla de emergencia (tabla 4).

\section{Tabla 4}

\section{Conocimiento del uso correcto de algunos métodos anticonceptivos}

\begin{tabular}{|l|c|c|c|c|}
\hline \multicolumn{1}{|c|}{ Conocimiento } & \multicolumn{2}{|c|}{ Enfermería } & \multicolumn{2}{c|}{ Derecho } \\
\hline \multicolumn{1}{|c|}{} & f & \% & f & \% \\
\hline Colocación correcta de preservativo & 97 & 97 & 187 & 93.5 \\
\hline $\begin{array}{l}\text { Cuándo tomar la pastilla de } \\
\text { emergencia }\end{array}$ & 97 & 97 & 94 & 47 \\
\hline Cuántas veces se utiliza la pastilla & 97 & 97 & 189 & 94.5 \\
\hline
\end{tabular}

Fuente: Cuestionario aspectos que intervienen en el uso de métodos anticonceptivos en estudiantes universitarios. $n=300$

La tabla 5 fue obtenida de varias preguntas del cuestionario Aspectos que intervienen en el uso de métodos anticonceptivos en estudiantes universitarios, en las cuales se podía proporcionar más de una respuesta. A su vez esta tabla demuestra que la principal fuente de información sobre los métodos anticonceptivos fueron los profesionales de la salud con $36.3 \%$; sin embargo, también muestra que otra fuente de información importante son los libros, revistas y medios de comunicación (impresos, electrónicos) con un 29\% del total de la muestra. 


\section{Tabla 5}

Principal fuente de información sobre MAC

\begin{tabular}{|c|c|c|c|c|}
\hline \multirow{2}{*}{ Fuentes de Información } & \multicolumn{2}{|c|}{ Enfermería } & \multicolumn{2}{|c|}{ Derecho } \\
\hline & $\mathbf{f}$ & $\%$ & $\mathbf{f}$ & $\%$ \\
\hline En su facultad & 94 & 45 & 98 & 30 \\
\hline Profesionales de salud & 46 & 22 & 63 & 19 \\
\hline $\begin{array}{l}\text { Libros, revistas y medios de comu- } \\
\text { nicación (impresos, electrónicos) }\end{array}$ & 18 & 9 & 69 & 21 \\
\hline Información recibida por los padres & 40 & 19 & 74 & 23 \\
\hline $\begin{array}{l}\text { Información proporcionada en la } \\
\text { religión }\end{array}$ & 11 & 5 & 22 & 7 \\
\hline Total de respuestas & 209 & 100 & 326 & 100 \\
\hline
\end{tabular}

Fuente: Cuestionario aspectos que intervienen en el uso de métodos anticonceptivos en estudiantes universitarios. $n=300$

La tabla 6 muestra los diferentes tipos de métodos anticonceptivos utilizados, donde se observa que el principal método anticonceptivo es el preservativo y en segundo lugar los anticonceptivos orales.

\section{Tabla 6}

\section{Métodos anticonceptivos más utilizado en el momento de la encuesta}

\begin{tabular}{|c|c|c|c|c|c|c|c|c|c|c|c|c|c|c|}
\hline \multirow{3}{*}{ Facultades } & \multicolumn{14}{|c|}{ Métodos anticonceptivos } \\
\hline & \multicolumn{2}{|c|}{ Ninguno } & \multicolumn{2}{|c|}{ Preservativo } & \multicolumn{2}{|c|}{$\begin{array}{l}\text { Anticoncep- } \\
\text { tivos orales }\end{array}$} & \multicolumn{2}{|c|}{$\begin{array}{l}\text { Implante } \\
\text { subdérmico }\end{array}$} & \multicolumn{2}{|c|}{$\begin{array}{c}\text { Dispositivo } \\
\text { intrauteri- } \\
\text { no (DIU) }\end{array}$} & \multicolumn{2}{|c|}{ Inyecciones } & \multicolumn{2}{|c|}{ Parches } \\
\hline & $f$ & $\%$ & $f$ & $\%$ & $f$ & $\%$ & $f$ & $\%$ & $f$ & $\%$ & $f$ & $\%$ & $f$ & $\%$ \\
\hline Enfermería & 32 & 32 & 52 & 52 & 2 & 2 & 2 & 2 & 4 & 4 & 7 & 7 & 1 & 1 \\
\hline Derecho & 43 & 21.5 & 115 & 57.5 & 21 & 10.5 & 5 & 2.5 & 3 & 1.5 & 9 & 4.5 & 4 & 2 \\
\hline
\end{tabular}

Fuente: Cuestionario aspectos que intervienen en el uso de métodos anticonceptivos en estudiantes universitarios. $n=300$

La tabla 7 contiene los datos sociodemográficos y los diferentes tipos de métodos anticonceptivos utilizados, la cual muestra que el principal método anticonceptivo utilizado es el preservativo y en segundo lugar son los anticonceptivos orales, las caracte- rísticas principales de estos usuarios son: edad entre 18 y 21 años, sexo indistinto, solteros y sin hijos. Los demás métodos son utilizados en menor proporción, por lo que se infiere que los jóvenes no se están protegiendo de una manera adecuada. 


\section{Tabla 7}

\section{Datos sociodemográficos y uso de métodos anticonceptivos}

\begin{tabular}{|c|c|c|c|c|c|c|c|c|c|c|c|c|c|c|}
\hline \multirow{3}{*}{$\begin{array}{l}\text { Datos so- } \\
\text { ciodemo- } \\
\text { gráficos }\end{array}$} & \multicolumn{14}{|c|}{ Métodos anticonceptivos } \\
\hline & \multicolumn{2}{|c|}{ Ninguno } & \multicolumn{2}{|c|}{ Preservativo } & \multicolumn{2}{|c|}{$\begin{array}{l}\text { Anticoncep- } \\
\text { tivos orales }\end{array}$} & \multicolumn{2}{|c|}{$\begin{array}{l}\text { Implante } \\
\text { subdérmico }\end{array}$} & \multicolumn{2}{|c|}{$\begin{array}{c}\text { Dispositivo } \\
\text { intrauteri- } \\
\text { no (DIU) }\end{array}$} & \multicolumn{2}{|c|}{ Inyecciones } & \multicolumn{2}{|c|}{ Parches } \\
\hline & $f$ & $\%$ & $f$ & $\%$ & $f$ & $\%$ & $f$ & $\%$ & $f$ & $\%$ & $f$ & $\%$ & $f$ & $\%$ \\
\hline $\begin{array}{ll}\text { Edad } & 18-21 \\
& 22-25\end{array}$ & $\begin{array}{l}64 \\
11\end{array}$ & $\begin{array}{c}21 \\
3\end{array}$ & $\begin{array}{c}126 \\
41\end{array}$ & $\begin{array}{l}42 \\
14\end{array}$ & $\begin{array}{l}12 \\
11\end{array}$ & $\begin{array}{l}4 \\
3\end{array}$ & $\begin{array}{l}3 \\
4\end{array}$ & $\begin{array}{c}1 \\
1.3\end{array}$ & $\begin{array}{l}4 \\
3\end{array}$ & $\begin{array}{c}1.3 \\
1\end{array}$ & $\begin{array}{l}7 \\
9\end{array}$ & $\begin{array}{c}2.3 \\
3\end{array}$ & $\begin{array}{l}4 \\
1\end{array}$ & $\begin{array}{l}1.3 \\
0.3\end{array}$ \\
\hline $\begin{array}{l}\text { Sexo Masc. } \\
\text { Femenino }\end{array}$ & $\begin{array}{l}12 \\
63\end{array}$ & $\begin{array}{c}4 \\
21\end{array}$ & $\begin{array}{l}78 \\
89\end{array}$ & $\begin{array}{l}26 \\
30\end{array}$ & $\begin{array}{c}2 \\
21\end{array}$ & $\begin{array}{l}0.6 \\
89\end{array}$ & $\begin{array}{l}1 \\
6\end{array}$ & $\begin{array}{c}0.3 \\
2\end{array}$ & $\begin{array}{l}0 \\
7\end{array}$ & $\begin{array}{c}0 \\
2.33\end{array}$ & $\begin{array}{c}2 \\
14\end{array}$ & $\begin{array}{l}0.6 \\
4.6\end{array}$ & $\begin{array}{l}2 \\
3\end{array}$ & $\begin{array}{c}0.6 \\
1\end{array}$ \\
\hline $\begin{array}{l}\text { Edo civil } \\
\text { Separado } \\
\text { Unión libre } \\
\text { Casado } \\
\text { Soltero }\end{array}$ & $\begin{array}{c}2 \\
0 \\
2 \\
71\end{array}$ & $\begin{array}{c}1 \\
0 \\
1 \\
24\end{array}$ & $\begin{array}{c}5 \\
4 \\
3 \\
155\end{array}$ & $\begin{array}{c}2 \\
1 \\
1 \\
52\end{array}$ & $\begin{array}{c}1 \\
2 \\
1 \\
19\end{array}$ & $\begin{array}{c}3 \\
1 \\
0.33 \\
6.3\end{array}$ & $\begin{array}{l}0 \\
1 \\
1 \\
5\end{array}$ & $\begin{array}{c}0 \\
0.33 \\
0.33 \\
1.66\end{array}$ & $\begin{array}{l}0 \\
2 \\
1 \\
4\end{array}$ & $\begin{array}{c}0 \\
0.66 \\
0.33 \\
1.33\end{array}$ & $\begin{array}{c}2 \\
0 \\
0 \\
14\end{array}$ & $\begin{array}{c}0.66 \\
0 \\
0 \\
4.6\end{array}$ & $\begin{array}{l}0 \\
0 \\
0 \\
5\end{array}$ & $\begin{array}{c}0 \\
0 \\
0 \\
1.66\end{array}$ \\
\hline Hijos $\begin{array}{r}\text { Sí } \\
\text { No }\end{array}$ & $\begin{array}{c}5 \\
70\end{array}$ & $\begin{array}{c}2 \\
23\end{array}$ & $\begin{array}{c}15 \\
152\end{array}$ & $\begin{array}{c}5 \\
51\end{array}$ & $\begin{array}{c}1 \\
22\end{array}$ & $\begin{array}{l}0.33 \\
7.33\end{array}$ & $\begin{array}{l}2 \\
5\end{array}$ & $\begin{array}{l}0.66 \\
1.66\end{array}$ & $\begin{array}{l}4 \\
3\end{array}$ & $\begin{array}{c}1.33 \\
1\end{array}$ & $\begin{array}{c}3 \\
13\end{array}$ & $\begin{array}{c}1 \\
4.3\end{array}$ & $\begin{array}{l}1 \\
4\end{array}$ & $\begin{array}{l}0.33 \\
1.33\end{array}$ \\
\hline
\end{tabular}

Fuente: Cuestionario aspectos que intervienen en el uso de métodos anticonceptivos en estudiantes universitarios. $n=300$

\section{Tabla 8}

Datos sociodemográficos y fuentes de información sobre MAC

\begin{tabular}{|c|c|c|c|c|c|c|c|c|c|c|c|c|}
\hline \multirow{3}{*}{$\begin{array}{c}\text { Datos } \\
\text { sociodemográficos }\end{array}$} & \multicolumn{12}{|c|}{ Fuentes de información } \\
\hline & \multicolumn{2}{|c|}{ Padres } & \multicolumn{2}{|c|}{ Hermanos } & \multicolumn{2}{|c|}{ Profesores } & \multicolumn{2}{|c|}{$\begin{array}{l}\text { Profesio- } \\
\text { nales de la } \\
\text { salud }\end{array}$} & \multicolumn{2}{|c|}{ Amigos } & \multicolumn{2}{|c|}{$\begin{array}{l}\text { Libros, revistasy } \\
\text { medios de com. (im- } \\
\text { presos y electrónicos) }\end{array}$} \\
\hline & $f$ & $\%$ & $f$ & $\%$ & $f$ & $\%$ & $f$ & $\%$ & $f$ & $\%$ & $f$ & $\%$ \\
\hline $\begin{array}{l}18-21 \\
22-25\end{array}$ & $\begin{array}{c}34 \\
8\end{array}$ & $\begin{array}{l}11.3 \\
2.6\end{array}$ & $\begin{array}{l}3 \\
1\end{array}$ & $\begin{array}{c}1 \\
0.3\end{array}$ & $\begin{array}{c}19 \\
8\end{array}$ & $\begin{array}{l}6.3 \\
2.6\end{array}$ & $\begin{array}{l}75 \\
34\end{array}$ & $\begin{array}{c}25 \\
11.3\end{array}$ & $\begin{array}{c}24 \\
7\end{array}$ & $\begin{array}{c}8 \\
2.3\end{array}$ & $\begin{array}{l}65 \\
22\end{array}$ & $\begin{array}{l}21.6 \\
7.3\end{array}$ \\
\hline $\begin{array}{l}\text { Masculino } \\
\text { Femenino }\end{array}$ & $\begin{array}{l}13 \\
29\end{array}$ & $\begin{array}{l}4.3 \\
9.6\end{array}$ & $\begin{array}{l}1 \\
3\end{array}$ & $\begin{array}{c}0.3 \\
1\end{array}$ & $\begin{array}{c}6 \\
21\end{array}$ & $\begin{array}{l}2 \\
7\end{array}$ & $\begin{array}{l}25 \\
84\end{array}$ & $\begin{array}{l}8.3 \\
28\end{array}$ & $\begin{array}{l}16 \\
15\end{array}$ & $\begin{array}{c}5.3 \\
5\end{array}$ & $\begin{array}{l}36 \\
52\end{array}$ & $\begin{array}{c}12 \\
17.3\end{array}$ \\
\hline $\begin{array}{r}\text { Separado } \\
\text { Unión libre } \\
\text { Casado } \\
\text { Soltero }\end{array}$ & $\begin{array}{c}1 \\
0 \\
0 \\
41\end{array}$ & $\begin{array}{c}0.3 \\
0 \\
0 \\
13.6\end{array}$ & $\begin{array}{l}0 \\
1 \\
0 \\
3\end{array}$ & $\begin{array}{c}0 \\
0.3 \\
0 \\
1\end{array}$ & $\begin{array}{c}0 \\
1 \\
1 \\
25\end{array}$ & $\begin{array}{c}0 \\
0.3 \\
0.3 \\
8.3\end{array}$ & $\begin{array}{c}3 \\
5 \\
3 \\
98\end{array}$ & $\begin{array}{c}1 \\
1.6 \\
1 \\
32.6\end{array}$ & $\begin{array}{c}1 \\
0 \\
2 \\
28\end{array}$ & $\begin{array}{c}0.3 \\
0 \\
0.6 \\
9.3\end{array}$ & $\begin{array}{c}5 \\
2 \\
2 \\
78\end{array}$ & $\begin{array}{l}1.6 \\
0.6 \\
0.6 \\
26\end{array}$ \\
\hline $\begin{array}{l}\text { Sí } \\
\text { No }\end{array}$ & $\begin{array}{c}7 \\
35\end{array}$ & $\begin{array}{c}2.3 \\
11.6\end{array}$ & $\begin{array}{l}0 \\
4\end{array}$ & $\begin{array}{c}0 \\
1.3\end{array}$ & $\begin{array}{c}3 \\
24\end{array}$ & $\begin{array}{l}1 \\
8\end{array}$ & $\begin{array}{l}12 \\
97\end{array}$ & $\begin{array}{c}4 \\
32.3\end{array}$ & $\begin{array}{c}3 \\
28\end{array}$ & $\begin{array}{c}1 \\
9.3\end{array}$ & $\begin{array}{c}6 \\
81\end{array}$ & $\begin{array}{c}2 \\
27\end{array}$ \\
\hline
\end{tabular}

Fuente: Cuestionario aspectos que intervienen en el uso de métodos anticonceptivos en estudiantes universitarios. $n=300$ 
En la tabla 8 se muestran los datos sociodemográficos y las principales fuentes de información acerca de los métodos anticonceptivos. La principal fuente de información en ambos grupos de edad, en solteros y sin hijos son los profesionales de la salud y en segundo lugar a través de los libros, revistas y medios de comunicación. Cabe destacar que la mayor parte de la población encuestada tiene estas características.

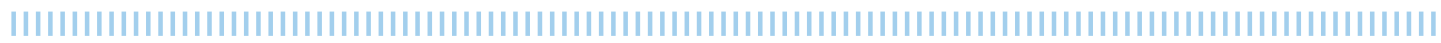

\section{Discusión}

En el estudio observamos que el sexo femenino prevaleció en un $67.7 \%$, esta cifra es similar a lo reportado por García y Alfaro, ${ }^{6}$ en donde los estudiantes universitarios fueron en mayor porcentaje del sexo femenino $(77 \%)$. El $91 \%$ de la población encuestada son solteros, observación semejante a lo referido por Mafla y Yamá, ${ }^{16}$ quienes mencionan que un $98 \%$ son solteros y sólo el $2 \%$ viven en unión libre. Respecto a los jóvenes en este estudio, el $72 \%$ tienen una vida sexual activa y el $89 \%$ no tienen hijos. Lo anterior explica que, a pesar de no tener pareja, los jóvenes universitarios tienen vida sexual activa y embarazos como consecuencia, por lo cual se hace necesaria la adecuada información acerca del debido uso de métodos anticonceptivos, ya que sólo el $58 \%$ de los encuestados refirió tener un buen conocimiento al respecto. La mayoría de los estudiantes de enfermería reciben información sobre métodos en su facultad con un $94 \%$, mientras que en los de derecho es menos de la mitad con un $49 \%$.

A pesar del alto porcentaje de estudiantes con vida sexual activa, el método más utilizado en la población es el preservativo con un $55.7 \%$, mientras que los demás métodos son pobremente utilizados. En cuanto al conocimiento de métodos, el $58.3 \%$ refirió que es bueno, $29.7 \%$ aceptable, $11 \%$ excelente y sólo el $1.5 \%$ indicó que es malo, sin embargo, no se ve reflejado en las respuestas posteriores, ya que el $5.3 \%$ no conoce el momento adecuado para la colocación del preservativo, 3\% no sabe cuándo ingerir la pastilla de emergencia y sólo el $69.9 \%$ sabe la indicación de uso de la misma, además sólo el $58.16 \%$ sabe las veces que se debe utilizar al año, ya que el utilizarlo más de 2 veces al año genera efectos adversos. Esto es muy similar a la estadística Nacional reportada en el 2014 por el Instituto Nacional de Estadística y Geografía (INEGI), la cual señala que $95 \%$ ha escuchado y conoce algún método anticonceptivo, pero el 37\% utiliza alguno de ellos en su primera relación sexual. Según Toro y Estrada, los jóvenes abusan de la píldora, porque la utilizan de forma inadecuada. Es importante que el uso de los métodos anticonceptivos sea acompañado de consejería.

La principal fuente de información de los estudiantes acerca de la salud sexual fue el personal de salud; sin embargo, esto sólo fue en un $36.3 \%$, seguido por los medios de comunicación con un $29 \%$. Esta última cifra no es adecuada dado que en muchas ocasiones la información recabada por este medio no es fidedigna y a veces ni siquiera es obtenida de fuentes científicas, lo que se refleja en prácticas de alto riesgo para su salud. El 38\% de los estudiantes refieren que la información proporcionada por parte de sus padres la consideran aceptable.

Se indagó acerca de la religión, ya que en el estudio de Mafla y Yamá, refiere que la religión influye en el uso de anticonceptivos. De la muestra, el $86.4 \%$ de los encuestados profesan alguna religión, pero sólo el $11 \%$ de los estudiantes refiere que la religión que profesan promueve el uso de métodos anticonceptivos, a través de pláticas o charlas educativas. Sin embargo, esta fuente no fue un medio de información común en nuestra muestra. 
En este contexto, la universidad debe ser un lugar que favorezca la formación integral y humanista en sus estudiantes, abierta a la libre discusión de las ideas en todos los campos del conocimiento. Paradójicamente, la educación sexual, no está integrada de modo formal en el sistema curricular y, sin embargo, cada vez es más necesario difundir conocimientos que logren cambiar ciertas actitudes sexistas. Es necesario procurar una buena educación sexual, porque, de acuerdo a nuestros resultados, es la principal fuente de información a este respecto, y debería estar muy por encima de otros canales de obtención de dicha información.

Dentro de la universidad encuestada, la clínica de Enfermería en Salud Interactiva (ENSAIN) fomenta la salud de los estudiantes, docentes y todo el personal que labora, fomentando estilos de vida saludables, motivando acciones personales y grupales para la promoción de la salud a través de ferias de salud que se organizan durante el ciclo escolar. Lo que lleva a pensar que existe un área de desaprovechamiento para el desempeño de estrategias en cuanto al uso de métodos anticonceptivos, siendo éstas, la Facultad de Enfermería y el programa de ENSAIN que tienen como misión brindar servicios de atención primaria en salud de alta calidad en casos de mediana complejidad, y ambulatorios promoviendo estilos de vida saludable en la población universitaria, propiciando con sus acciones mejorar la calidad de vida de los alumnos de la institución. Se debe reforzar la información en cuanto al uso de métodos anticonceptivos, ocupando el plan curricular con el aprovechamiento del desempeño de los estudiantes en curso, fomentando así, mayor desarrollo de prácticas y experiencias profesionales en cuanto a primer nivel en materia de salud comunitaria, para así evitar embarazos no deseados y enfermedades de transmisión sexual. En este punto, la enfermería juega un papel fundamental en el conocimiento y uso de nuevos mé- todos anticonceptivos, debe orientar a los jóvenes en su toma de decisiones a través de la clasificación de tipos de métodos que se puedan acoplar a cada joven. ${ }^{17}$

Para la utilización correcta de un método anticonceptivo se deben conocer las características, grado de efectividad, ventajas, desventajas, efectos adversos e inclusive las creencias de cada joven, esto es necesario para que el método permita alcanzar las metas reproductivas en forma efectiva y segura para la salud y de acuerdo con la situación de vida. En casos especiales, se recomienda usar dos métodos anticonceptivos para tener mayor protección contra el embarazo e ITS. ${ }^{2}$

Por lo tanto, nuestros resultados deberán servir para desarrollar intervenciones de salud reproductiva en el ámbito universitario, además puede dar cabida a la propuesta de realizar modificaciones en los contenidos curriculares agregando talleres sobre el tema de métodos anticonceptivos con la finalidad de sensibilizar sobre la importancia del uso de métodos, ya que hoy en día las consecuencias de no utilizarlos se consideran como un problema de la salud pública, debido al incremento de embarazos no deseados, ITS y deserción escolar en los adultos jóvenes.

Finalmente es oportuno que se continúe con esta línea de investigación, y que se analicen los aspectos generales y específicos aquí planteados sobre el uso de métodos anticonceptivos, llevar a cabo un estudio correlacional, así como la política educativa en promoción a la salud, para lo cual se podrían considerar las siguientes sugerencias a tomar en cuenta:

A Realizar una promoción más extensa sobre métodos anticonceptivos, y dar más información al personal y a los estudiantes, acerca del método que les convendría utilizar y que no perjudique su salud.

- Crear instrumento para valorar el 
grado de conocimiento de los jóvenes estudiantes, después de haber tomado un taller sobre métodos anticonceptivos.

- Realizar ferias de la salud donde el tema principal sean los métodos anticonceptivos.

- Capacitar a los docentes sobre métodos anticonceptivos para crear a través de ellos una conciencia en los estudiantes sobre su salud sexual.

- Capacitar sobre la educación sexual, dar una idea de las actitudes, de las presiones, conciencia de las alternativas y sus consecuencias. Debe aumentar el amor, el conocimiento propio, debe mejorar la toma de decisiones y la técnica de la comunicación.

\section{Conclusiones}

A pesar de que los profesionales de la salud son la principal fuente de información de los jóvenes, sólo el $36.3 \%$ lo reciben por este medio. Esto se refleja en las deficiencias de conocimiento al respecto de métodos anticonceptivos. Por lo tanto, es un área de oportunidad para que el personal de enfermería informe sobre los métodos anticonceptivos, sobre todo entre sus estudiantes.

\section{Bibliografía}

1. Gamboa M.C. y Gutiérrez S.M. Métodos anticonceptivos glosario, antecedentes, marco jurídico, políticas públicas y recomendaciones internacionales. [Internet]. (2015). Available from: http://www.diputados.gob.mx/sedia/sia/spi/SAPI-ISS-71-15.pdf.

2. Norma oficial mexicana De los servicios de planificación familiar (modificada). Secretaria de salud, publicación, diario oficial de la federación. [Internet]. Norma Oficial Mexicana NOM-005SSA2-1993. [Internet]. Available from: http://www. dof.gob.mx/nota_detalle.php?codigo $=676842 \& \mathrm{fec}$ ha $=21 / 01 / 2004$.

3. Tecuapetla, M. E. Conocimiento de métodos anticonceptivos en adolescentes. (Tesis de Licenciatura. Universidad Nacional Autónoma de México). [Internet]. (2012). Available from: http://132.248.9.195/ ptd2013/Presenciales/0702850/0702850.pdf.

4. Organización Mundial de la Salud (OMS). Departamento de Salud Reproductiva e Investigaciones Conexas. Recomendaciones sobre prácticas seleccionadas para el uso de anticonceptivo. [Internet]. 2018;(3). Available from: https://apps.who.int/iris/ bitstream/handle/10665/259814/9789243565408spa.pdf; jsessionid $=$ C868E42B0B8D9F8F1168B3137 93B5A92? sequence $=1$

5. Castro, P.M., Peniche, M.A y Peña, W.J. Conocimientos, mitos y prácticas sobre el uso de métodos anticonceptivos asociados al incremento de embarazos no planificados en estudiantes adolescentes en dos instituciones educativas en la ciudad de Cartagena. Facultad de ciencias de la salud programa de enfermería. Cartagena. [Internet] 2012:45-47. Available from: http://siacurn.curnvirtual.edu.co:8080/xmlui/ bitstream/handle/123456789/577/conocimientos, $\% 20$ mitos $\% 20 y \% 20$ practicas $\% 20$ sobre $\% 20$ e $1 \% 20$ uso $\% 20$ de $\% 20 \mathrm{~m} \%$ c3\% 89todos $\%$ 20anticonceptivo.pdf? seq uence $=1$.

6. García, A. y Alfaro, A. Sexualidad y anticoncepción en jóvenes universitarios de Albacete. Revista de enfermería. [Internet] 2014;14(3). Available from: https://www.uclm.es/ab/enfermeria/revista/numero\% 2014/sexualidad y anticoncepci\%F3n_en_j. htm.

7. Gracia, H. Deserción Universitaria en México. Milenio. [Internet] (2015). de Available from: http://www. milenio.com/firmas/maximiliano_gracia_hernandez/ Desercion-universitaria-Mexico_18_559324103. html

8. Moreno, T.B., Arellano, J.G., Colin, A.G., Lomas, S.A. y Sánchez R.L. Deserción de Estudiantes Universitarios por embarazo. Revista electrónica de investigación en enfermería FESI-UNAM. [Internet]. 2013;2(4). Available from: http://journals.iztacala.unam. $\mathrm{mx} /$ index.php/cuidarte/article/viewFile/261/290

9. Romero, S. T. Relación entre el nivel de conocimiento y la frecuencia del uso de métodos anticonceptivos por 
los adolescentes. (Tesis para obtener el diploma de especialista en medicina familiar, Universidad Nacional Autónoma de México. Facultad de medicina, división de estudios de posgrado investigación). [Internet]. 2012. Available from: http://132.248.9.195/ ptd2013/febrero/089627646/Index.html.

10. Anzalone, L. y Mattera, A. Infecciones de transmisión sexual. Temas de bacteriología y virología médica: Instituto de Higiene Universidad de la 81República. [Internet]. 2015;(2):227-243. Available from: http://www.higiene.edu.uy/cefa/2008/infeccionestrasmitidassexualmente.pdf

11. Organización Mundial de la Salud (OMS). Infecciones de transmisión sexual. Nota descriptiva (110) [Internet]. 2016. Available from: http://www.who. int/mediacentre/factsheets/fs110/es/.

12. Instituto Nacional de Estadística y Geografía (INEGl). Estadísticas a propósito del día internacional de la juventud (15 a 29 años) 12 de agosto, datos nacionales. [Internet]. 2016. Available from: http:// www.inegi.org.mx/saladeprensa/aproposito/2016/ juventud2016 0.pdf

13. Instituto Nacional de Estadística y Geografía (INEGI). Estadísticas a propósito del día internacional de la juventud 12 de agosto, datos nacionales. [Internet]. 2015. Available from: http://www.inegi.org.mx/ saladeprensa/aproposito/2015/juventud0.pdf

14. Encuesta Nacional de Salud y Nutrición (ENASUT). Instituto Nacional de Salud Pública. [Internet]. 2012. Available from: http://ensanut.insp.mx/

15. Gamarra, T. Factores asociados que influyen en el inicio de actividad sexual en adolescentes escolares de Villa San Francisco- Santa Anita, Lima- Perú 2009 (tesis de maestría). Universidad Nacional Federico Villarreal, Lima, Perú. 2009

16. Mafla, P. y Yamá, C. Factores sociales, culturales y religiosos que impiden la utilización de métodos anticonceptivos en mujeres en edad fértil en el servicio de ginecología del hospital Marco Vinicio, iza de la provincia de sucumbíos en el periodo marzo-agosto 2014. (Tesis de licenciatura, Universidad Politécnica Estatal del Carchi). [Internet]. 2014. Available from: https://es.scribd.com/document/306755109/44factores-sociales-culturales-y-religiosos-que-impiden-la-utilizacion-de-metodos-anticonceptivos-enmujeres-en-edad-fertil-en-el-servicio-de-gineco

17. Enfermería en Salud Interactiva (ENSAIN) Hernández, H.T Universidad Autónoma de Querétaro. Facultad de Enfermería, Campus San Juan del Río. Base de datos 2017. https://docs.google.com/ spreadsheets/d/1kMp4_Zmk5nOUx1dEpAyw-ntY zUQLbIKJsmjOvxyr4AQ/edit?usp=drive_web\&ou id $=116449505296043602526$. 